\title{
Batraciens nouveaux du Cameroun
}

\author{
par \\ Jean-Luc PERRET \\ Institut de Zoologie de l'Université de Neuchâtel (Suisse) \\ et Foulassi (Cameroun).
}

Avec 6 figures dans le texte

J'ai donné ailleurs ${ }^{1}$ la diagnose de Phrynobatrachus werneri hylaios Perret et présente ici la description détaillée et critique de cette nouvelle forme. D'autre part, j'ai découvert et observé ces derniers mois une espèce d'Hyperolius que je crois nouvelle et dont je donne plus loin la description. Je remercie vivement mes collègues J. Guibé de Paris et R. Laurent d'Elisabethville de leurs précieuses informations et des comparaisons de matériel faites à ma demande.

\section{Phrynobatrachus werneri hylaios Perret}

Holotype: 1 đૈ Foulassi, Cameroun; alt. 710 m, fôrèt. Capturé le 12.1.1959. Muséum de Genève, no 964.100.

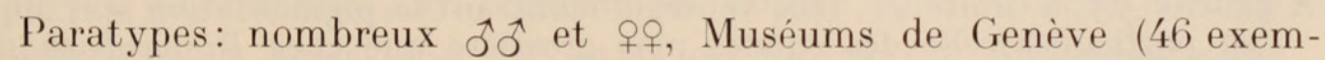
plaires), Paris et Londres, capturés de 1954 à 1959.

Diagnose: Petite espèce (21 $\mathrm{mm}$ de long.) forme forestière de Phrynobatrachus werneri (Nieden), de moyenne et basse altitude, se distinguant de la forme typique par les caractères suivants:

1 Bull. Soc. neuchâtel. Sci. nat., 82, 1959

Rev. Suisse de Zool., T. 66, 1959 


\begin{tabular}{|c|c|}
\hline werneri hylaios (fig. 1) & werneri werneri \\
\hline $\begin{array}{l}\text { 1. Forme svelte. } \\
\text { 2. Largeur de la tête } 6-7 \mathrm{~mm} \text {. } \\
\text { 3. Largeur du tibia } 2,8-3 \mathrm{~mm} \text {. } \\
\text { 4. Plis latéraux du sac vocal, fai- } \\
\text { blement développés, peu pro- } \\
\text { fonds, souvent avec quelques } \\
\text { plis secondaires subparallèles. } \\
\text { 5. Gorge des đđđ verruqueuse sur } \\
\text { toute la surface. }\end{array}$ & $\begin{array}{l}\text { Forme robuste. } \\
8-9 \mathrm{~mm} \text {. } \\
3,5 \mathrm{~mm} \text {. } \\
\text { Plis latéraux du sac vocal, forte- } \\
\text { ment développés, profonds, } \\
\text { sans plis secondaires. } \\
\text { Gorge des ôô lisse, sauf au men- } \\
\text { ton et à l'extérieur des plis } \\
\text { latéraux du sac vocal. }\end{array}$ \\
\hline
\end{tabular}

Description: Forme svelte. Une papille linguale présente. Museau assez pointu mais à bout tout de même arrondi, plus long que l'orbite, dépassant la mandibule. Canthus rostralis appa-

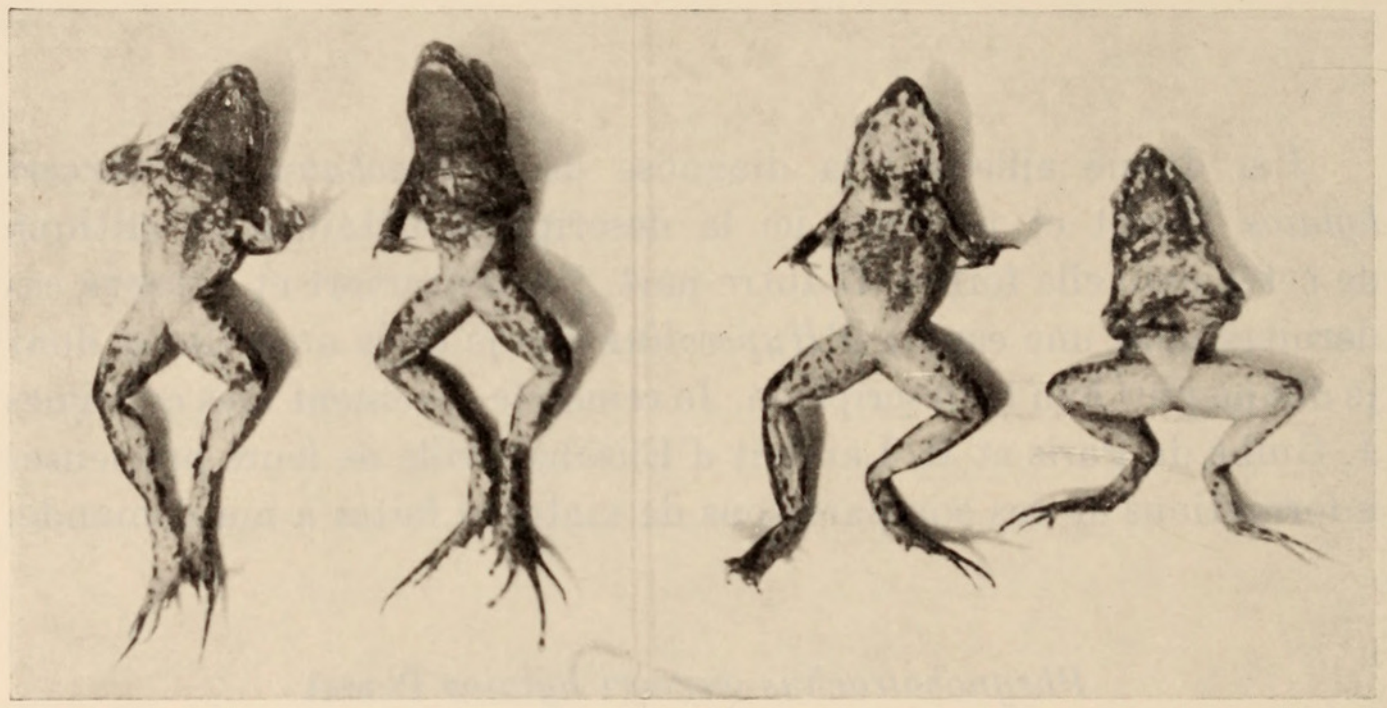

FIG. 1.

Phrynobatrachus werneri hylaios Perret. 1/1.

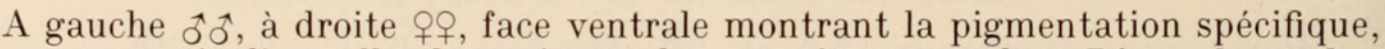
en particulier celle des cuisses plus grossière que chez Dimorphognathus africanus (fig. 4) dont les jeunes lui ressemblent beaucoup extérieurement.

rent mais non anguleux. Région loréale concave. Tête plus longue que large. Narines à peine plus près du bout du museau que de l'œil. Tympan peu visible, mesurant la moitié du diamètre oculaire. Espace interorbitaire plus grand que la largeur d'une paupière supérieure. 
Doigts terminés par des petits disques qui ont à peu près le même diamètre que ceux des orteils. Palmure postérieure faiblement développée, laissant libres au quatrième orteil 4 phalanges ou au moins trois trois-quart (fig. 2). Un tubercule métatarsien interne allongé, mesurant la moitié ou un plus de la moitié du premier orteil. Un tubercule métatarsien externe petit, ovoïde, plus proche du tubercule métatarsien interne que du tubercule tarsien (3e tubercule pédieux). Celui-ci, petit, est prolongé par une arête en forme de virgule en direction du tubercule métatarsien interne.

L'articulation tibio-tarsienne atteint le milieu de l'œil.

Peau lisse sur la tête et le haut du dos, parsemée de petites verrues mousses sur le bas du dos et les côtés. Ventre, poitrine et membres lisses dessous tandis que la gorge est couverte de verru-cosités chez le $\widehat{o}$ (holotype) et lisse chez la $q$ (paratype). Ces petites verrues gu-
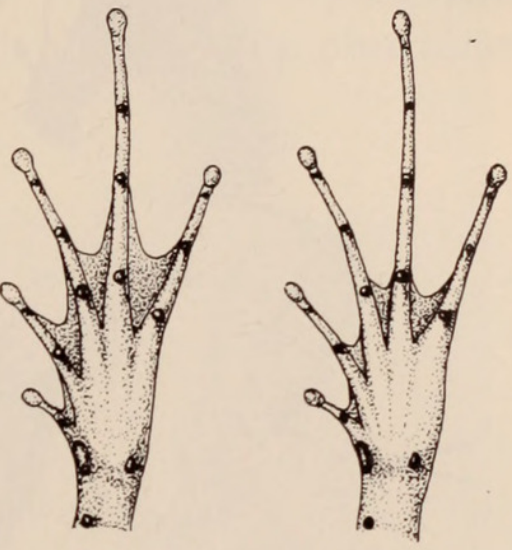

FIg. 2.

Pied de Phrynobatrachus ogoensis Boul. (à gauche) et de Phrynobatrachus werneri hylaios Perret (à droite) montrant le développement de la palmure. laires du $\widehat{\jmath}$, vues à la loupe, apparaissent claires sur fond noir; elles sont peu visibles à l'œil nu. Plis en ) (, caractéristique du genre, présents. Ils commencent derrière l'œil et, s'étendent sur le tiers du dos environ.

Coloration: Dos gris-brun avec une barre foncée surlignée de clair entre les yeux (comme chez beaucoup d'autres espèces du genre) et une autre tache entre les plis glandulaires en ) (. Côté de la tête brun foncé; cette coloration sombre, limitée en arrière par le pli postoculaire, se termine en pointe sous le bras comme chez plicatus et batesi (fig. 3), mais en moins vif. Membres avec des barres foncées dessus. Lèvres foncées, plus ou moins tachetées de blanc. L'alternance de taches claires et foncées sur la mandibule est une ornementation qui se retrouve chez plusieurs espèces de Phrynobatrachus et chez Dimorphognathus africanus (fig. 4). Gorge, poitrine et une bonne partie du ventre tacheté (qo) ou envahi ( $\widehat{\jmath})$ de brun violacé (fig. 1). A part une faible zone supérieure claire, les cuisses sont assez grossièrement tachetées de 
foncé (pigmentation moins étendue, plus périphérique et plus fine chez werneri werneri). Tibias tachetés sur leur pourtour.

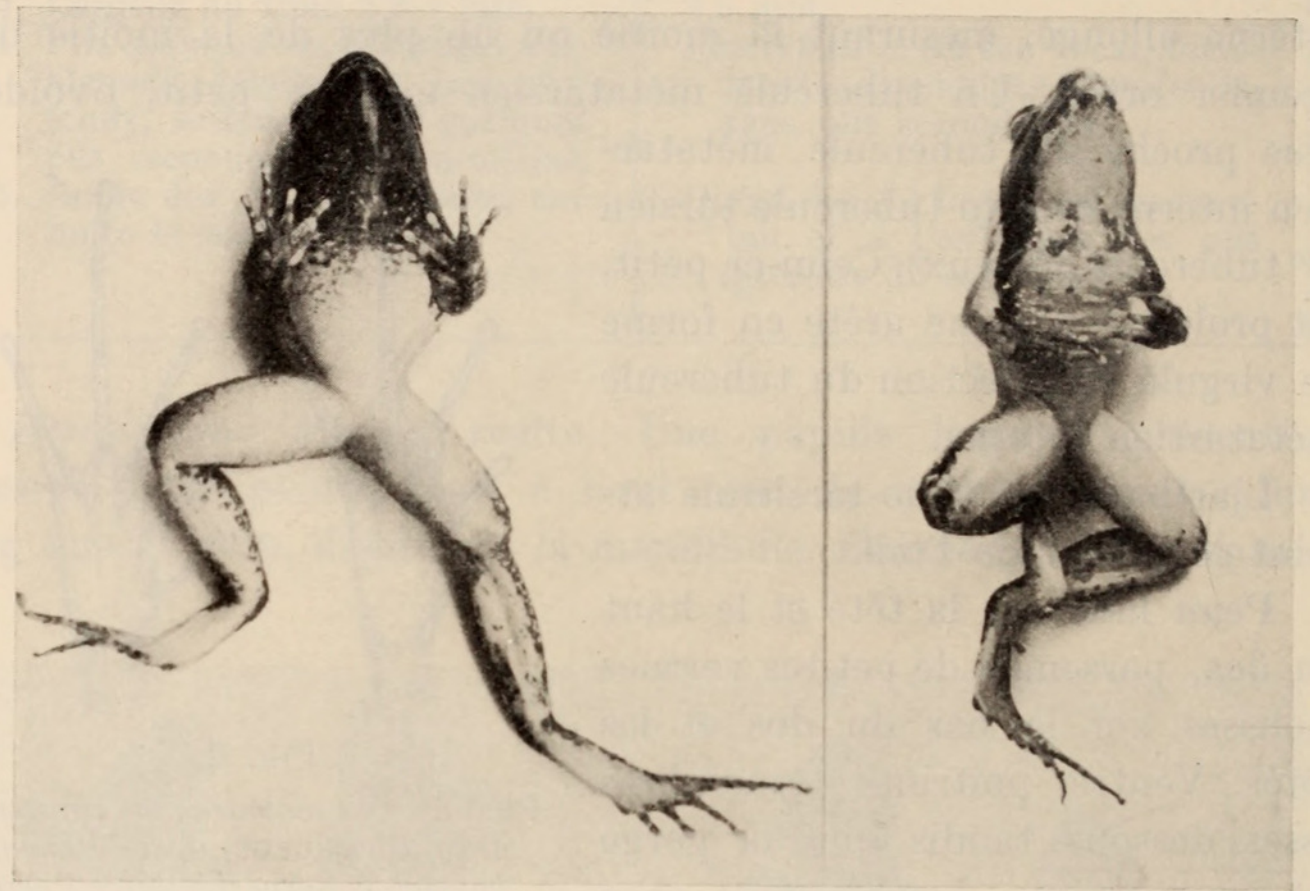

FIG. 3.

Phrynobatrachus batesi (Boul.). 1/1.

A gauche $\hat{o}$, à droite $\rho$, face ventrale montrant la pigmentation spécifique. Les cuisses immaculées permettent de distinguer cette espèce des autres Phrynobatrachus forestiers du Cameroun.

Mensurations en $\mathrm{mm}$

\begin{tabular}{|c|c|c|c|}
\hline & Holotype ot & Paratype + & $\begin{array}{l}\text { moyenne } \\
\delta^{*} \delta^{0} \text { et } \text { if }\end{array}$ \\
\hline 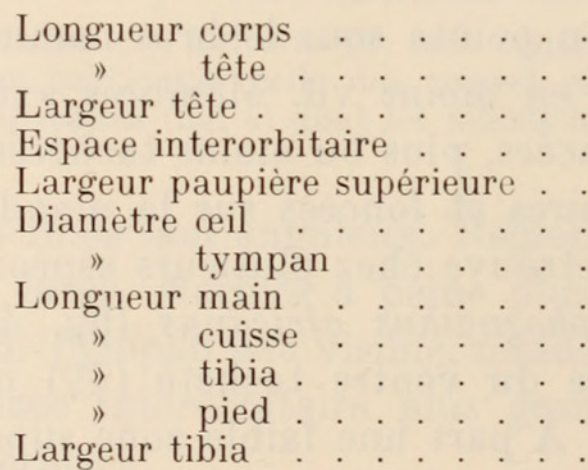 & $\begin{array}{c}21 \\
8 \\
7 \\
2 \\
1,5 \\
2,8 \\
1,5 \\
5,6 \\
9 \\
10 \\
14,7 \\
3\end{array}$ & $\begin{array}{l}21,5 \\
9 \\
7 \\
2 \\
1,5 \\
3 \\
1,5 \\
6 \\
10,3 \\
10,8 \\
15,5 \\
3\end{array}$ & $\begin{array}{l}21 \\
8,75 \\
7\end{array}$ \\
\hline
\end{tabular}


Phrynobatrachus werneri hylaios est une forme répandue dans les marigots de la région forestière du sud Cameroun. Elle vit sur le sol humide où règne constamment une demi-obscurité à cause de la luxuriance de la végétation. A toute heure du jour ou de la nuit, on entend le faible cri des $\widehat{o} \widehat{o}$ rappelant plus des vibrations d'élytres qu'un chant de batracien, un bruit de crécelle très atténué. Dans le même biotope se trouve Dimorphognathus africanus dont le cri, un "crouââ» nasillard, beaucoup plus sonere, s'entend de temps à autre.
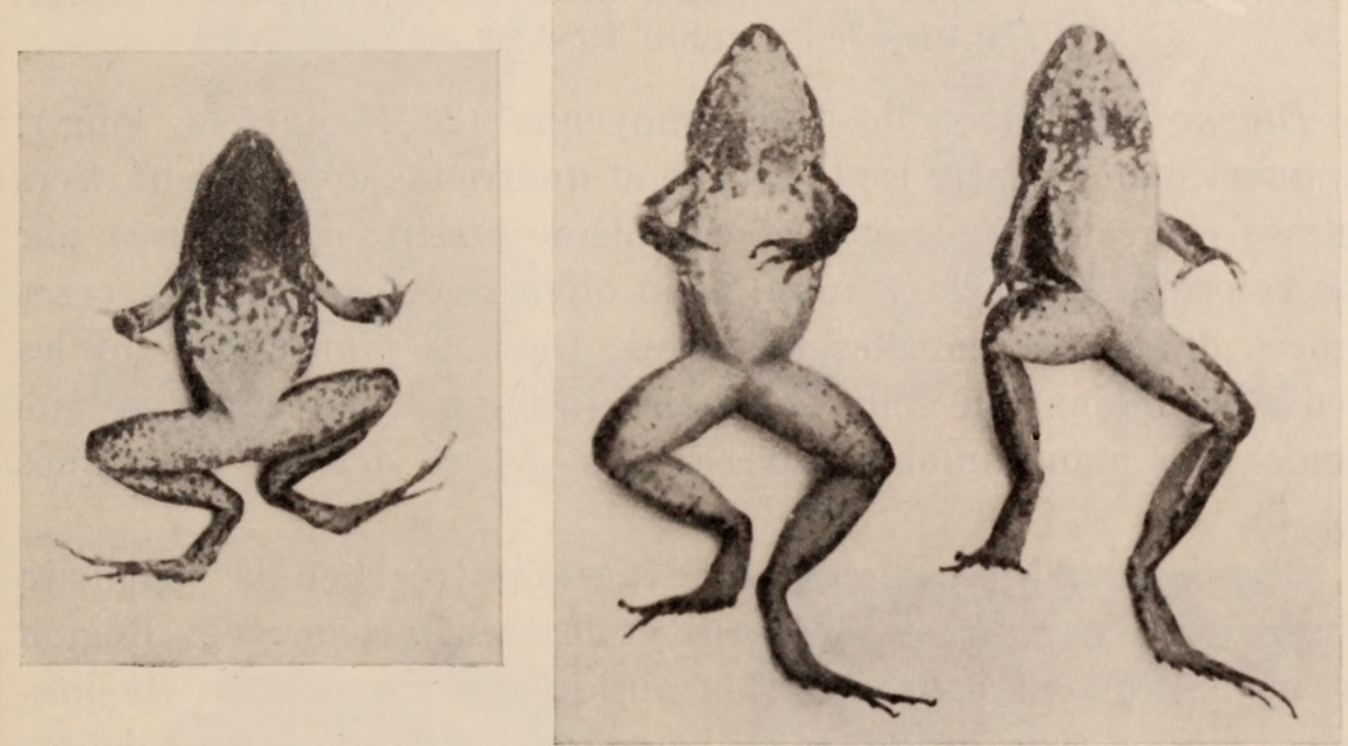

FIG. 4.

Dimorphognathus africanus (Hallowell). 1/1.

A part les pseudo-dents mandibulaires, cette espèce a tout à fait le faciès d'un Phrynobatrachus. Elle vit à côté d'hylaios.

Je décris Phrynobatrachus werneri hylaios après l'avoir observé pendant plus de quatre ans. J'ai d'abord cru avoir affaire à ogoensis Boul. décrit du Gabon voisin mais qui possède une palmure nettement plus développée, (fig. 2); puis à de jeunes batesi (Boul.) qui pourraient être facilement confondus, mais qui diffèrent par leurs cuisses immaculées inférieurement (fig. 3). La forme adulte de batesi (30 $\mathrm{mm}$ ) étant en outre notablement plus grande que celle d'hylaios $(21 \mathrm{~mm})$. Enfin, j'ai capturé en savane et en forêt élevée la forme typique werneri werneri qui vivante, se distingue très nettement d'hylaios tout en paraissant être en relation subspécifique avec lui. 
Parmi les formes voisines par la taille et la palmure réduite, les types de graueri (Nieden), brevipalmatus (Ahl) et gutturosus Chabanaud ont pu être comparés à hylaios et trouvés distincts. Enfin, dispar (Boulenger) a été signalé dans le groupe des espèces à glandes fémorales, absentes chez hylaios.

\section{Hyperolius mosaicus n. sp.}

Holotype: 1 q Ngam/Sangmelima, Cameroun; alt. 710. m, forêt. Capturé le 10.3.1959. Muséum de Genève no 965.12.

Paratypes: 1 q et 8 ๙ิ ô, Muséum de Genève no 965.13 à 965.21. Capturés en octobre 1958 et en mars 1959.

Diagnose: Espèce de taille moyenne (24-27 mm de long.); un quart palmée entre les troisième et quatrième doigts, deux tiers palmée entre les quatrième et cinquième orteils; caractérisée par une coloration dorsale sombre, brun olivâtre ornée de zones ocres semées de perles blanches émaillées. Dessous jaune vif sauf les pieds, les mains qui sont noirs ainsi qu'une tache anale et une bande sous-mandibulaire. Livrées des $\widehat{o} \widehat{o}$ et des qq identiques (fig. 5).

Description: Museau arrondi dépassant très peu la mâchoire inférieure. Canthus rostralis obtus et légèrement incurvé. Région loréale légèrement concave. Tête aussi longue que large (la longueur mesurée du museau à l'angulaire). Palmure des mains, entre le troisième et le quatrième doigt, s'étendant jusqu'au premier tubercule sous-articulaire du troisième doigt et, par une frange, jusqu'à la base du disque du quatrième doigt. La palmure du pied, au troisième orteil du côté externe, atteint presque la base du disque (soit 1 phalange libre); au quatrième orteil, du côté interne elle arrive entre les deux tubercules sous-articulaires distaux (soit 2,5 phalanges libres); du côté externe, elle atteint le tubercule distal (soit 2 phalanges libres); enfin au cinquième orteil, elle s'étend presque jusqu'au disque terminal (soit 1 phalange libre).

Coloration (en vie): Parties supérieures avec un fond brun olivâtre, les palmures des mains et des pieds noires, l'extrémités des doigts et des orteils, particulièrement les disques terminaux, jaunes. Un triangle céphalique, deux zones arrondies scapulaires paramédianes et une large zone dorsale jaune ocre se détachent 
du fond et sont plus ou moins semées de perles blanches émaillées. Le nombre et la grosseur des perles varient; elles sont rares ou font défaut sur la tête. Chez un paratype tout le dos est envahi de perles; chez un autre, au contraire, on ne compte qu'une seule perle dans toute la région scapulaire (fig. 5). Parties inférieures d'un jaune vif sauf les mains et les pieds qui sont noirs jusqu'au niveau de la palmure, les extrémités des doigts et des orteils étant
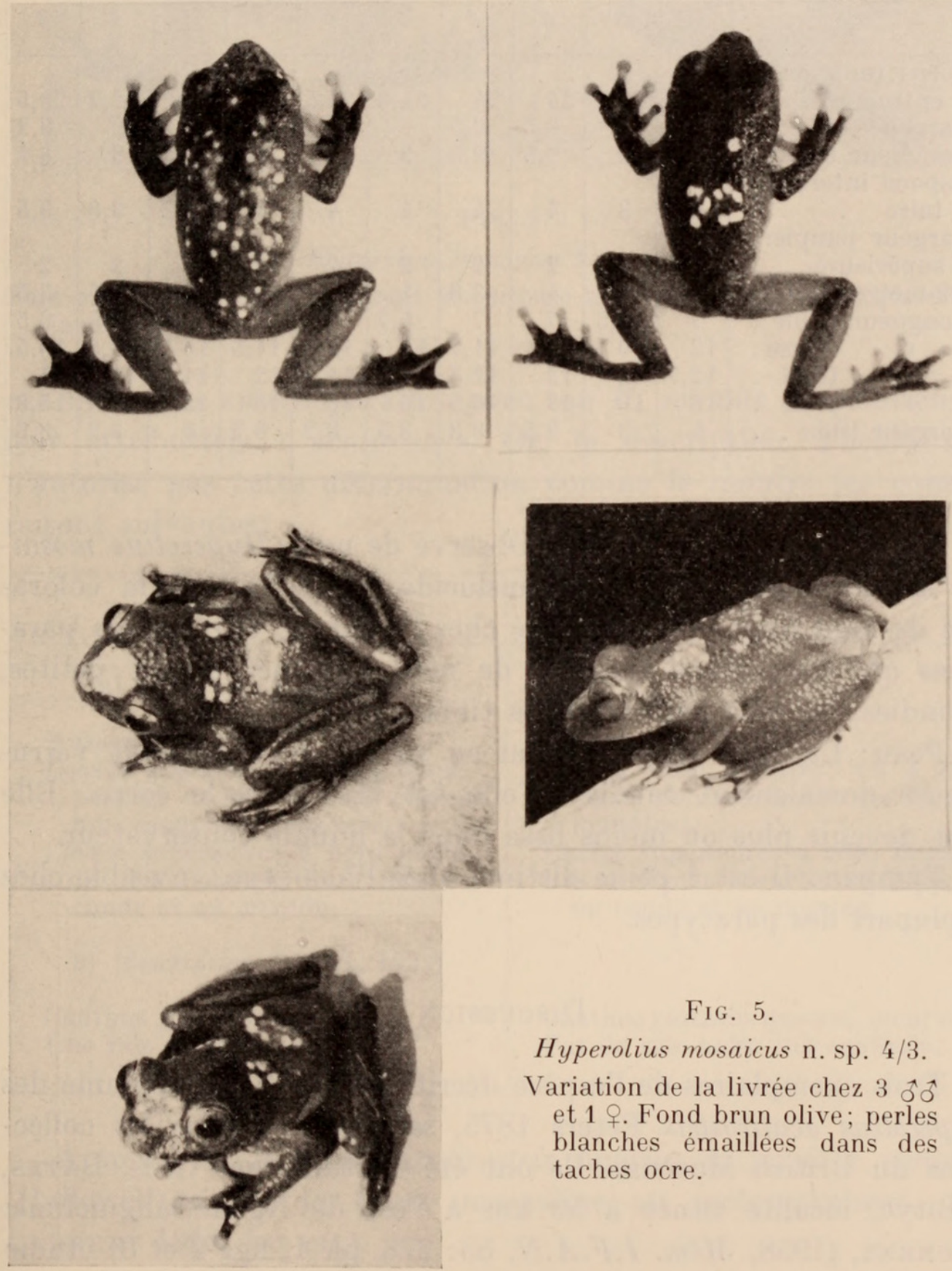

FIG. 5.

Hyperolius mosaicus n. sp. 4/3.

Variation de la livrée chez 3 $\widehat{0} \widehat{o}$ et 1 q. Fond brun olive; perles blanches émaillées dans des taches ocre. 
jaunes. Il y a encore des petites bandes ou des taches noires à la périphérie des tibias et des cuisses, notamment une forte tache subanale et enfin une zone marginale sous-mandibulaire (fig. 6).

Mensurations en $\mathrm{mm}$

\begin{tabular}{|c|c|c|c|c|c|c|c|c|c|c|}
\hline & $\begin{array}{l}\text { Holo- } \\
\text { type }\end{array}$ & \multicolumn{9}{|c|}{ Paratypes } \\
\hline & $q$ & † juv. & o & o & o & o & o & $0^{*}$ & ô & o \\
\hline Corps (museau & & & & & & & & & & \\
\hline entrejambe) & 27 & 22 & 24 & 24 & 24,3 & 24,5 & 24 & 23 & 22,7 & 23,5 \\
\hline Largeur tête & 10 & 9 & 9,5 & 9,5 & 9 & 9 & 8,5 & 8,5 & 9 & 8,2 \\
\hline Longueur tête : & 10 & 9 & 9,5 & 9,5 & 9 & 9 & 8,5 & 8,5 & 9 & 8,2 \\
\hline $\begin{array}{c}\text { Espace interorbi- } \\
\text { taire }\end{array}$ & 4 & 3 & 4 & 4 & 4 & 4 & 4 & 35 & 38 & 35 \\
\hline Largeur paupière & & & & & & & & & & \\
\hline supérieure & 2 & 1,7 & 2 & 2 & 2 & 2 & 2 & 2 & 2 & 2 \\
\hline Diamètre de l'œil & 4 & 3 & 4 & 3,8 & 4 & 4 & 4 & 3,8 & 3,7 & 3,5 \\
\hline Longueur main . & 7 & 6,5 & 7 & 7 & 6,7 & 7 & 7 & 6,5 & 6,5 & 6,5 \\
\hline " cuisse. & 12 & 10 & 11 & 11,7 & 11 & 10,8 & 11,5 & 10,5 & 10,5 & 10,5 \\
\hline tibia. & 12,7 & 12 & 12 & 12,5 & 11,5 & 11,3 & 12 & 11 & 11,2 & 11 \\
\hline " pied. & 18 & 16 & 17 & 16,5 & 16 & 16 & 16 & 15,5 & 15,8 & 15,8 \\
\hline Largeur tibia. . & 4 & 3 & 3,5 & 3,8 & 3,5 & 3,2 & 3,3 & 3 & 3,2 & 3,5 \\
\hline
\end{tabular}

Variation de la coloration: Observé de nuit, Hyperolius mosaicus apparait jaune olivâtre translucide. Dans l'alcool, la coloration de fond n'est plus uniforme chez l'holotype et quelques paratypes qui sont bruns tachetés de noir. Les taches sont petites, arrondies, les plus nettes sur les tibias.

Peau: La peau chez l'animal en vie est régulièrement verruqueuse dorsalement tandis qu'elle est lisse sous le corps. Elle peut devenir plus ou moins lisse dans le liquide conservateur.

Tympan: Il est à peine distinct chez l'holotype, invisible chez la plupart des paratypes.

\section{Discussion}

Trois exemplaires de l'espèce décrite ici, considérés comme des Hyperolius acutirostris Peters 1875, se trouvent dans les collections du British Museum. Ils ont été capturés par G. L. Bates, à Bitye, localité située à $50 \mathrm{~km}$ à l'est de Ngam/Sangmelima. Laurent, (1958, Mém. I.F.A.N. 53: 278, pl. 1, figs 2 et 3) étudie 


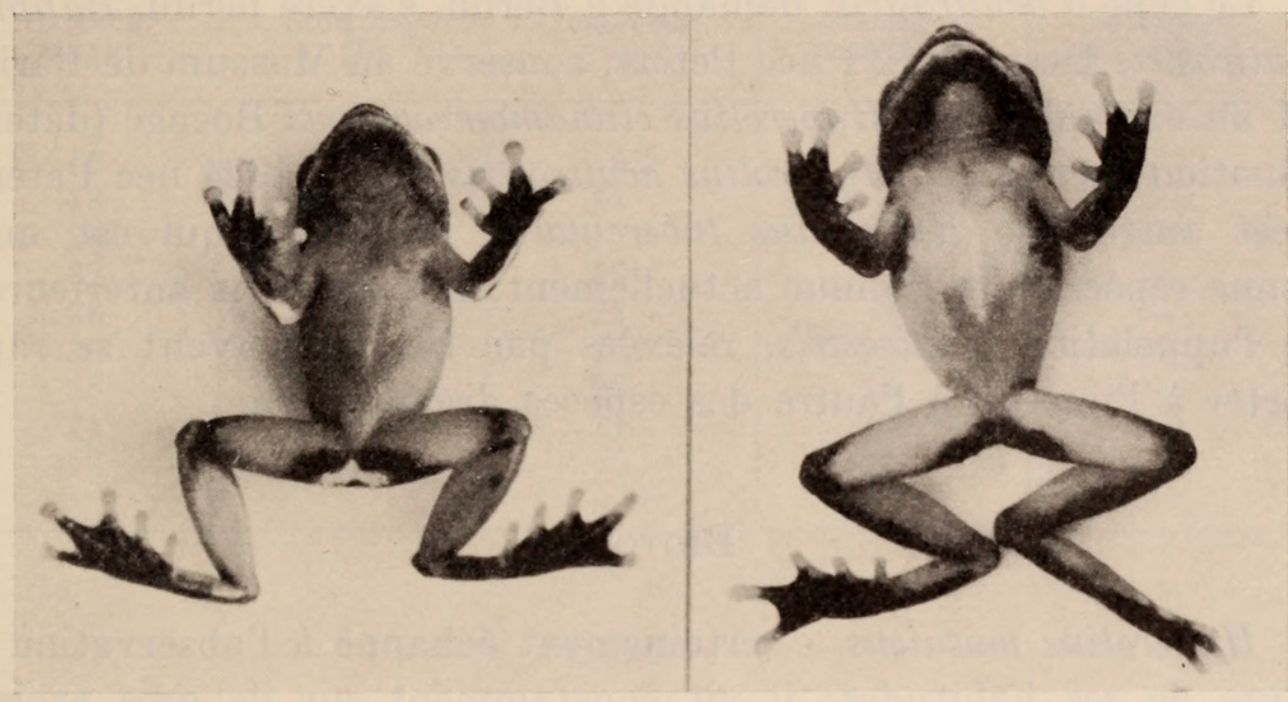

FIG. 6.

Hyperolius mosaicus $\mathrm{n}$. sp. 4/3.

Face ventrale, $\sigma^{\hat{t}}$ et $q$, montrant la curieuse mélanisation périphérique. Fond jaune vif.

ces spécimens en comparaison avec son $H$. zonatus en maintenant leur attribution à acutirostris. Or, la description d'acutirostrsi n'autorise pas cette détermination comme le montre les comparaisons suivantes:

\begin{tabular}{|c|c|}
\hline acutirostris Peters & \multicolumn{1}{|c|}{ mosaicus n. sp. } \\
\hline a) (description originale) & \\
$\begin{array}{c}\text { Museau pointu. } \\
\text { Dessus gris-bleu; vu à la loupe, } \\
\text { ponctué de bleu avec un réseau } \\
\text { noir (donc sur fond noir). }\end{array}$ & $\begin{array}{c}\text { Museau arrondi } \\
\text { Dessus brun olivâtre; vu à la } \\
\text { loupe, ponctué de noir sur fond } \\
\text { jaunâtre. }\end{array}$ \\
$\begin{array}{c}\text { Lèvres supérieures à bord blanc. } \\
\text { Une tache blanche au talon, au } \\
\text { coude et au menton. }\end{array}$ & $\begin{array}{c}\text { Pas de tache blanche au talon, } \\
\text { au coude et au menton. }\end{array}$ \\
b) (description de AHL, 1931) & \\
$\begin{array}{c}\text { Canthus rostralis aigu, droit } \\
\text { Une raie noire sub-canthale. }\end{array}$ & $\begin{array}{l}\text { Canthus rostralis mousse, incurvé. } \\
\text { Pas de raie noire sub-canthale. }\end{array}$ \\
\hline
\end{tabular}

A mon avis, le type d'acutirostris suggère un jeune $c$. concolor (Hallowell) ayant la livrée masculine en métamorphose. (Cf. Laurent 1958, loc. cit.). 
Le type d'acutirostris, demandé à Berlin, s'avère perdu. Rappia acutirostris Despax 1911 nec Peters, conservé au Museum de Paris, est un exemplaire de Hyperolius cinnamomeoventris Bocage (détermination Laurent). Hyperolius acutirostris Noble 1924 nec Peters n'est autre que Hyperolius tuberculatus Mocquard qui est une bonne espèce, bien connue actuellement. Les citations antérieures de l'appelation acutirostris, relevées par NoвLe doivent se rapporter à l'une ou à l'autre des espèces discutées ici.

\section{BiоTоPе}

Hyperolius mosaicus a certainement échappé à l'observation à cause de ses habitudes: il vit constamment sur de gros arbres où il se nourrit, pont et se reproduit sans descendre obligatoirement au sol. Comme les trous et les cavités pouvant conserver l'eau de pluis sont moins rares vers le bas du tronc qu'en hauteur, particulièrement chez les essences à contreforts ailés, on peut y rencontrer des rassemblements de cette petite rainette à l'apparition des premières pluies. Ce biotope est également celui d'Acanthixalus spinosus (Buchholz et Peters) que j'ai bien observé ces dernières années.

\section{Biologie}

La ponte de une a deux dizaines d'œufs est déposée dans une masse gélatineuse transparente, collée au tronc juste au-dessus du niveau de l'eau. L'incubation dure de 13 à 20 jours. Les larves tombées à l'eau se nourrissent de débris végétaux morts, de larves d'insectes et des cadavres de leur propre espèce. La métamorphose dure environ trois mois coincidant avec la saison des pluies. La fin de celles-ci entrainant souvent l'assèchement du iřou.

Le têtard est robuste, à tête large et queue bien musclée. La nageoire dorsale qui ne dépasse pas beaucoup le corps en hauteur a des bords subparallèles et se termine assez brusquement. Il est régulièrement pigmenté sauf au ventre; le corps est sombre, la queue plus claire. Le bec est finement denticulé; la formule dentaire, $\frac{1}{1+\frac{1}{2}}$ est constante. Je donne, à la suite un tableau résumant la croissance du têtard. 


\begin{tabular}{|c|c|c|c|c|}
\hline $\begin{array}{c}\text { Longueur } \\
\text { totale }\end{array}$ & $\begin{array}{c}\text { Longueur } \\
\text { tête }\end{array}$ & $\begin{array}{c}\text { Longueur } \\
\text { queue }\end{array}$ & $\begin{array}{c}\text { Membres } \\
\text { postérieurs }\end{array}$ & $\begin{array}{c}\text { membres } \\
\text { antérieurs }\end{array}$ \\
\hline & 6,8 & 9,2 & - & - \\
16 & 7 & 11 & - & - \\
28 & 11 & 17 & 1,7 & - \\
32 & 13 & 19 & 3,5 & - \\
38 & 14 & 24 & 2 & - \\
36 & 19 & 17 & 10 & 9 \\
35 & 12 & 22 & 16 & 8 \\
24 & 13 & 11 & 17 & \\
16 & 11 & 6 & 15 & \\
\hline
\end{tabular}

\section{Chant}

Hyperolius mosaicus émet un ou plusieurs petits coups de sifflet de tonalité moyenne: «ou-uh... ou-uh» qui ne portent pas très loin. Tous les Hyperolius ont un cri flûté plus ou moins sonore. Il faut une oreille très exercée pour distinguer les espèces du genre simplement d'après le timbre. A côté de mosaicus, on peut trouver communément, perchés à faible hauteur, ocellatus guttatus, steindachneri pardalis, cinnamomeoventris, tuberculatus, platyceps lombaris. 


\section{$2 \mathrm{BHL}$ Biodiversity Heritage Library}

1959. "Batraciens nouveaux du Cameroun." Revue suisse de zoologie 66, 711-721. https://doi.org/10.5962/bhl.part.117921.

View This Item Online: https://www.biodiversitylibrary.org/item/149483

DOI: https://doi.org/10.5962/bhl.part.117921

Permalink: https://www.biodiversitylibrary.org/partpdf/117921

\section{Holding Institution}

American Museum of Natural History Library

\section{Sponsored by}

BHL-SIL-FEDLINK

\section{Copyright \& Reuse}

Copyright Status: In copyright. Digitized with the permission of the rights holder.

Rights Holder: Muséum d'histoire naturelle - Ville de Genève

This document was created from content at the Biodiversity Heritage Library, the world's largest open access digital library for biodiversity literature and archives. Visit BHL at https://www.biodiversitylibrary.org. 